\section{Conclusions du colloque}

\author{
Pierre-Benoît Joly
}

IFRIS, Université Paris Est-LATTS, 5 , boulevard Descartes, Champs-sur-Marne, 77454 Marne-la-Vallée Cedex 2, France.

joly@inra-ifris.org
Ma conclusion sera brève. La journée a été riche et passionnante et je me garderai de tenter de la résumer. Je remercie Pierre Tambourin pour sa présence et sa disponibilité. Grâce à sa volonté d'initier ce partenariat entre Genopole ${ }^{\circledR}$ et I'IFRIS, nous disposons d'un espace pour travailler ensemble à une meilleure compréhension de la science en société et à une amélioration du dialogue entre les sciences du vivant et les sciences humaines et sociales.

Je souhaite également remercier les organisateurs des ateliers qui ont joué un rôle essentiel dans la construction de ce colloque. Je remercie tous les intervenants pour la très grande qualité de leurs présentations. Je remercie enfin Nicole Chémali et son équipe sans qui ce colloque n'aurait pas eu lieu.

Après un colloque de ce type, nous partons nécessairement avec plus de questions que de réponses. Cependant, nous avons indéniablement progressé dans notre questionnement. Ce colloque sur les transformations des modes de production des connaissances dans les sciences du vivant illustre magnifiquement la nécessité d'aborder ces questions à partir de différentes perspectives et à différentes échelles. Pour analyser les transformations conjointes des contenus épistémiques, des pratiques de recherche, des formes d'organisation, de la circulation et de l'appropriation des connaissances, il est nécessaire de croiser les regards des biologistes et ceux des chercheurs en sciences sociales. II est aussi nécessaire de faire varier les échelles, du laboratoire à la communauté scientifique ou à l'échelle nationale ou internationale, mais aussi de resituer les changements les plus contemporains dans une histoire longue.
Voici les enseignements principaux que j'ai pu tirer de ce colloque.

- Depuis le lancement des grands programmes de séquençage des génomes à la fin des années 1980, une biologie à haut débit s'est progressivement constituée. L'automatisation et la robotisation des instruments d'analyse, la création de plates-formes, la constitution de grands consortiums... bref, tout un ensemble de dispositifs instrumentaux et organisationnels ont changé fondamentalement les modes d'acquisition de données dans les sciences du vivant. Première implication, on assiste à un véritable « déluge informationnel ». Non seulement la quantité de données disponibles ne cesse d'augmenter mais le phénomène s'accélère, créant une obsolescence rapide dans de nombreux champs de recherche. Deuxième implication, l'organisation de la recherche en réseaux hybrides, associant biologistes, cliniciens, bio-informaticiens, chercheurs académiques, entreprises, utilisateurs... devient la règle, ce qui ne manque pas d'interroger sur l'évolution des normes qui régissent ces champs hybrides. Quelles sont les implications de ces changements sur les contenus épistémiques ? Assiste-t-on au passage d'une recherche basée sur la formulation d'hypothèses à une recherche basée sur le traitement de données de masse ? À la fin du réductionnisme, dont la substitution des approches centrées sur les gènes par des approches centrées sur des réseaux serait l'une des manifestations? Les interventions et les débats qui ont suivi ont montré qu'il faut se garder d'une fascination pour la nouveauté et que, si les changements sont de grande ampleur, les implications d'un point de vue épistémologique ne sont pas forcément dans les oppositions évoquées mais plutôt dans des inflexions moins visibles a priori, comme par exemple le changement du statut et du rôle des modèles, les nouvelles convergences entre analyses fonctionnelles et approches évolutionnistes.

- Les différentes interventions ont aussi démontré l'intérêt de l'étude de la production de connaissances scientifiques dans leur contexte économique, politique et social. L'exemple de l'histoire des recherches sur les gènes BRCAl et BRCA2 et des analyses des conflits sur les 
brevets illustre parfaitement cette idée. En effet, ce domaine scientifique ne relève pas simplement de transformations épistémiques et de questions qu'il serait possible de traiter uniquement sous un angle économique. Les discussions sur l'utilisation des tests génétiques de prédisposition au cancer du sein montrent que cette transformation sous-tend la création d'un marché, d'un système social et de formes de régulation de la production de connaissances. Le cas du gène $B R C A I$ permet de comprendre l'importance des transformations, notamment en matière de brevets. L'analyse historique joue ici un rôle déterminant. Il est important d'analyser les mondes sociaux associés aux changements des recherches sur le vivant en adoptant une approche systématique des règles de droit, des normes, des pratiques mais aussi des régimes discursifs.

- L'analyse des « communs » scientifiques et des « communs » des ressources génétiques et biologiques invite à dépasser la dichotomie classique entre bien public et bien privé. Afin de limiter les problèmes qui peuvent découler d'un excès d'exclusivisme lié aux brevets, les acteurs inventent des arrangements qui permettent de partager les connaissances et de faire circuler les ressources. Les communs sont désormais très répandus mais leur diversité est encore trop méconnue. Les recherches en droit et en sciences sociales ont joué un rôle très important dans cette émergence ; elles jouent un rôle essentiel dans leur connaissance et peuvent contribuer très utilement à une diffusion rapide des bonnes pratiques.
La prochaine conférence portera probablement sur la biologie de synthèse. Nous avons évoqué au cours de ce colloque l'importance de ce domaine émergent pour la production de connaissances en biologie : compte tenu de la complexité des organismes vivants, la construction artificielle d'organismes est probablement l'un des moyens privilégiés pour comprendre les processus biologiques. Ce projet est au cœur de la biologie de synthèse qui recèle aussi d'importants enjeux économiques, sociaux et éthiques. En France, le champ de la biologie de synthèse se structure, au gré d'initiatives des chercheurs, des entreprises et des pouvoirs publics. Genopole ${ }^{\circledR}$ constitue l'un des principaux pôles de recherche en biologie de synthèse en France. À la suite du rapport de l'IFRIS, le Ministère de la recherche a demandé au CNAM de mettre en place un observatoire de la biologie de synthèse. Il est donc très opportun de dédier notre prochain colloque à ce sujet. $\diamond$
TIRÉS À PART

P.B. Joly 